\title{
不同结构烷基铝催化异戊二烯齐聚与聚合行为研究
}

\author{
彭伟戚佩瑶董凯旋贺爱华* \\ (山东省烯烃催化与聚合重点实验室 橡塑材料与工程教育部重点实验室 青岛科技大学高分子科学与工程学院
}

青岛 266042)

\begin{abstract}
摘要 烷基铝( $\mathrm{AlR}_{3}$ )作为 Ziegler-Natta 催化剂体系的助催化剂组分, 起到烷基化、还原主金属化合物、参与活性中心形 成与演变、链转移剂等重要作用. 然而烷基铝自身对二烯烃单体也具有催化作用. 本工作采用不同结构烷基铝如三乙 基铝 $\left(\mathrm{AlEt}_{3}\right.$ )、三异丁基铝 $\left(\mathrm{Al}(i-\mathrm{Bu})_{3}\right)$ 、氢化二异丁基铝 $\left(\mathrm{AlH}(i-\mathrm{Bu})_{2}\right)$ 、一氯二乙基铝 $\left(\mathrm{AlEt}{ }_{2} \mathrm{Cl}\right)$ 、二氯一乙基铝 $\left(\mathrm{AlEtCl}_{2}\right)$, 研 究了烷基铝的种类和浓度对异戊二烯催化行为的影响. 采用核磁共振氢谱 $\left.{ }^{1} \mathrm{H} N \mathrm{NR}\right)$ 、凝胶渗透色谱(GPC)、气相色谱质谱联用(GC-MS)等对产物的微观结构(顺式-1,4-和反式-1,4-含量)和分子量及分布等进行了表征，探讨了不同结构烷 基铝的催化行为. 发现烷基铝不仅可以催化异戊二烯齐聚, 与微量水作用后还可以引发异戊二烯阳离子聚合, 得到顺 反混合结构的线性聚合物. 烷基铝浓度对其催化行为有较大影响. 当 $n(\mathrm{Al}) / n(\mathrm{M})=1050 \times 10^{-5}$ 时, $\mathrm{AlEtCl}_{2}$ 的催化活性显 著提高, 产物主要为线性聚合物; 而其他结构烷基铝的催化活性较低. 当 $n(\mathrm{Al}) / n(\mathrm{M}) \leqslant 350 \times 10^{-5}$, 烷基铝自身催化异戍 二烯齐聚及聚合能力极弱. 过低和过高的烷基铝浓度都不利于获得高分子量聚合物. 这为深入理解 Ziegler-Natta 催化 剂体系烷基铝组分的催化作用及其对聚合物的影响提供依据.
\end{abstract}

关键词 烷基铝; 异成二烯; 催化; 齐聚; 聚合行为

\section{Oligomerization and Polymerization of Isoprene Catalyzed by Alkylaluminium with Different Structures}

\author{
Peng, Wei Qi, Peiyao Dong, Kaixuan He, Aihua*
}

(Shandong Provincial Key Laboratory of Olefin Catalysis and Polymerization, Key Laboratory of Rubber-Plastics (Ministry of Education), School of Polymer Science and Engineering, Qingdao University of Science and Technology, Qingdao 266042, China)

Abstract Alkylaluminium $\left(\mathrm{AlR}_{3}\right)$, as co-catalyst component in Ziegler-Natta catalytic system, plays important roles in the alkylation, forming and changing the structure and concentration of active centers through the reduction and reversible adsorption-desorption reactions with the metal compound of the catalyst, acting as chain transfer agent, etc. However, the alkylaluminium itself do have the catalytic effect on the conjugated diene monomers. In this article, alkylaluminium with different structures such as triethylaluminium $\left(\mathrm{AlEt}_{3}\right)$, triisobutylaluminium $\left(\mathrm{Al}(i-\mathrm{Bu})_{3}\right)$, diisobutylaluminium hydride $\left(\mathrm{AlH}(i-\mathrm{Bu})_{2}\right)$, diethylaluminium chloride $\left(\mathrm{AlEt}_{2} \mathrm{Cl}\right)$, ethylaluminium dichloride $\left(\mathrm{AlEtCl}_{2}\right)$ were used to catalyze isoprene oligomerization and polymerization. The effects of the structure and concentration of alkylaluminiums $\left(n(\mathrm{Al}) / n(\mathrm{M})=7 \times 10^{-5}, 35 \times 10^{-5}, 350\right.$ $\times 10^{-5}, 1050 \times 10^{-5}$ ) on the catalytic behaviors of isoprene were studied. The microstructure (trans-1,4 and cis-1,4), molecular weight and molecular weight distribution of the products were characterized by ${ }^{1} \mathrm{H}$ nuclear magnetic resonance spectroscopy ( ${ }^{1} \mathrm{H}$ NMR), gel permeation chromatography (GPC) and gas chromatography-mass spectrometry (GC-MS). It was found that alkylaluminium could initiate oligomerization and cationic polymerization of isoprene under the minor incorporation of $\mathrm{H}_{2} \mathrm{O}$, which were affected greatly by the structure and concentration of alkylaluminium. Using $\mathrm{AlEtCl}_{2}$ led to the highest catalytic activity and produced products containing more linear polymers with mixed cis-1,4/trans-1,4 structures when $n(\mathrm{Al}) / n(\mathrm{M})=1050 \times 10^{-5}$. The $\mathrm{Al}(i-\mathrm{Bu})_{3}$ and $\mathrm{AlH}(i-\mathrm{Bu})_{2}$ didn't have basically cation initiation ability, which led to isoprene oligomerization. The alkylaluminium with $n(\mathrm{Al}) / n(\mathrm{M}) \leqslant 350 \times 10^{-5}$ had negligible influence on the isoprene polymerization and oligomerization. And lower or higher alkylaluminium concentration were not beneficial to obtain polyisoprene with high molecular weight. The catalytic mechanism of alkylaluminium on isoprene was discussed, which provided a further understanding on the catalytic behavior of alkylaluminium components in Ziegler-Natta catalyst and the effect of alkylaluminium on polymers.

Keywords alkylaluminium; isoprene; catalysis; oligomerization; polymerization behavior

\footnotetext{
*E-mail: ahhe@qust.edu.cn; aihuahe@iccas.ac.cn

Received July 30, 2020; published October 9, 2020.

Project supported by the Major Scientific and Technological Innovation Project of Shandong Province (No. 2019JZZY010352) and Taishan Scholar Program. 项目受山东省重大科技创新项目(No. 2019JZZY010352)及泰山学者工程资助.
} 


\section{1 引言}

烷基铝作为 Ziegler-Natta 催化体系中不可缺少的助 催化剂, 具有十分重要的作用 ${ }^{[1-2]}$ : (1) $\mathrm{TiCl}_{4}$ 与烷基铝发 生烷基化反应，生成烷基钢和 $\mathrm{AlR}_{2} \mathrm{Cl} 、 \mathrm{AlRCl}_{2}$ 等副产物; （2）烷基钛发生均裂和还原反应, 生成 $\mathrm{Ti}^{3+}$ 或 $\mathrm{Ti}^{2+}$; (3) 反应过程中产生的自由基可以发生偶合或歧化终止等. Cavallo 等 ${ }^{[3]}$ 通过密度泛函理论(DFT)模拟了催化剂中位 于 $\mathrm{MgCl}_{2}$ (110)晶面的 $\mathrm{TiCl}_{4}$ 与 $\mathrm{AlEt}_{3}$ 发生烷基化反应的 过程, 认为反应包括 $\mathrm{AlEt}_{3}$ 的乙基与 $\mathrm{Ti}$ 原子配位、形成 过渡四元环和 $\mathrm{Al}-\mathrm{Et}$ 键断裂等过程. Terano 等 ${ }^{[4-6]}$ 指出烷 基铝在 Ziegler-Natta 催化剂活性中心立构选择性的产 生、转变中起着重要作用. Potapov 等 ${ }^{[7-8]}$ 证实 $\mathrm{AlR}_{2} \mathrm{Cl}$ 可 以吸附配位在催化剂表面. 范志强等 ${ }^{[9]}$ 也发现 $\mathrm{AlEt}_{2} \mathrm{Cl}$ 既可配位在活性中心 $\mathrm{Ti}$ 原子上, 降低活性中心数目和 催化剂的活性, 又可吸附在活性中心 $\mathrm{Ti}$ 原子周围的 $\mathrm{Mg}$ 原子上, 形成高全同定向性的活性中心, 还可与某些无 活性的二价铁配位, 形成新的活性中心. 因此烷基铝及 其与 $\mathrm{TiCl}_{4}$ 反应形成的副产物 $\mathrm{AlR}_{2} \mathrm{Cl}$ 及 $\mathrm{AlRCl}_{2}$ 等对 $\mathrm{Z}-\mathrm{N}$ 催化剂的活性和立构选择性有至关重要的影响 ${ }^{[10-15]}$.

20 世纪 50 年代以来, 许多质子酸或路易斯酸(如 $\mathrm{TiCl}_{4} 、 \mathrm{AlCl}_{3}$ 和 $\mathrm{AlEtCl}_{2}$ 等)作为阳离子引发剂, 催化异成 二烯、丁二烯聚合成粉状、粘稠状或油状产物 ${ }^{[16-19]}$, 温 度、时间和溶剂等因素对聚合物结构有很大影响 ${ }^{[20-22]}$, 产物中同时存在环状结构和线型结构(主要为 1,4-和 3,4结构 $)^{[23]}$. Gaylord 等 ${ }^{[24]}$ 发现 $\mathrm{TiCl}_{4} 、 \mathrm{AlCl}_{3}$ 和 $\mathrm{AlEtCl}_{2}$ 等路 易斯酸在正庚烷中催化异戊二烯阳离子聚合转化率较 低, 聚合物以环状结构为主, 线状结构的含量一般不超 过 $20 \%$, 但在芳香族溶剂中反应速率更高, 且聚合物中 线性结构含量增大. 当使用 $\mathrm{TiCl}_{4}-\mathrm{Al}\left(\mathrm{C}_{2} \mathrm{H}_{6}\right)_{2} \mathrm{X}(\mathrm{X}=\mathrm{F}, \mathrm{Br}$, $\mathrm{Cl}$ ) 催化异戊二烯聚合时, 其聚合活性高于单独使用 $\mathrm{AlR}_{2} \mathrm{X}$ 或 $\mathrm{AlRX}_{2}$, 聚合物以环状结构为主 ${ }^{[25]}$. Gaylord 等 ${ }^{[25]}$ 认为该反应的活性中心是 $\mathrm{TiCl}_{4}$ 与 $\mathrm{AlEtX}_{2}$ 反应产物 解离出的阳离子, 并提出了可能的环聚机理. Matyska 等 ${ }^{[26]}$ 发现在没有水或其他助剂的情况下，二氯乙基铝 在室温下催化异戊二烯的聚合速度极其缓慢, 微量的水 对催化剂的活性有积极影响. 这一发现揭示了二氯乙基 铝有催化活性的先决条件是少量的共催化物质(如水)的 存在.

1971 年, Uchida 等 ${ }^{[27]}$ 报道了 $\mathrm{VO}(\mathrm{OEt})_{3}-\mathrm{AlEt}_{3}$ 体系催 化异戊二烯可合成液体聚合物, $\mathrm{VO}(\mathrm{OEt})_{3}-\mathrm{AlEtCl}_{2}$ 体系 催化异成二烯环聚为不溶性聚合物. Akutagawa 等 ${ }^{[28]}$ 用 镍系催化剂 $\left(\mathrm{Ni}\left(\eta^{3}-\mathrm{C}_{3} \mathrm{H}_{5}\right)_{2}-\mathrm{PPh}\left(\mathrm{NEt}_{2}\right)_{2}, \quad \mathrm{Ni}\left(\eta^{3}-\mathrm{C}_{3} \mathrm{H}_{5}\right)_{2}-\right.$ $\mathrm{PPh}\left(\mathrm{NMe}_{2}\right)_{3}, \mathrm{Ni}\left(\eta^{3}-\mathrm{C}_{3} \mathrm{H}_{5}\right)_{2}-\mathrm{As}(i-\mathrm{Pr})_{3}$ 等)选择性地催化异 戊二烯聚合得到各种线性或环状齐聚物. 沈国良和于士 君等 ${ }^{[29-31]}$ 发现采用 $\mathrm{TiCl}_{4}-\mathrm{AlEt}_{1.5} \mathrm{Cl}_{1.5}$ 催化体系, 异戊二 烯易发生环三聚反应, 环三聚体的收率和选择性较高; 当采用 $\mathrm{TiCl}_{4}-\mathrm{Al}\left(\mathrm{C}_{2} \mathrm{H}_{5}\right)_{2} \mathrm{Cl}-\mathrm{Al}\left(\mathrm{C}_{2} \mathrm{H}_{5}\right) \mathrm{Cl}_{2}$ 催化体系, 低聚物 收率比单一使用 $\mathrm{Al}\left(\mathrm{C}_{2} \mathrm{H}_{5}\right)_{2} \mathrm{Cl}$ 或 $\mathrm{Al}\left(\mathrm{C}_{2} \mathrm{H}_{5}\right) \mathrm{Cl}_{2}$ 作助催化
剂高. 因此提出烷基铝首先将 $\mathrm{Ti}^{4+}$ 还原为 $\mathrm{Ti}^{3+}, \mathrm{Ti}^{3+}$ 与烷 基铝配位形成活性中心，两分子异戊二烯在 $\mathrm{Ti}$ 上配位 形成双 $\pi$ 配合物，当第三个异戊二烯分子插入 $\mathrm{Ti}-\mathrm{C}$ 键 末端，置换活性中心后形成三聚体.

Ziegler-Natta 催化体系中, 由于烷基铝在与主催化 剂的反应过程中形成了不同的烷基氯化物，且这些不同 结构的烷基铝副产物会直接影响 Ziegler-Natta 催化体系 形成的产物的结构与组成. 因此, 本工作研究了不同结 构烷基铝(如 $\mathrm{AlEt}_{3} 、 \mathrm{Al}(i-\mathrm{Bu})_{3} 、 \mathrm{AlH}(i-\mathrm{Bu})_{2} 、 \mathrm{AlEt}_{2} \mathrm{Cl}$ 、 $\mathrm{AlEtCl}_{2}$ ) 的单组分催化剂和烷基铝的浓度对异戊二烯聚 合行为的影响, 通过对产物的分析, 深入理解 Ziegler-Natta 催化剂体系中烷基铝组分的阳离子引发作 用产生的影响.

\section{2 结果与讨论}

\section{1 不同烷基铝的催化行为}

不同烷基铝在不同反应条件下催化异成二烯聚合 的实验数据及产物的表征结果汇总于表 1 中.

图 1 为 $30{ }^{\circ} \mathrm{C}$ 下不同烷基铝催化异戊二烯聚合的转 化率对比图. 当烷基铝与单体的物质的量比 $n(\mathrm{Al}) / n(\mathrm{M})$ $\leqslant 350 \times 10^{-5}$ 时, 各烷基铝催化异戊二烯的转化率极低, 只得到痕迹量的产物. 提高 $n(\mathrm{Al}) / n(\mathrm{M})$ 有利于单体转化 率的提高, 但实际降低了烷基铝的催化活性(即单位时 间 $1 \mathrm{~h}$ 内 $1 \mathrm{~mol}$ 烷基铝产生的聚合物(g.P/mol.Al/h))(表 1 ), 说明高烷基铝浓度下也只有一部分烷基铝产生活性物 种并引发异戊二烯聚合. 当 $n(\mathrm{Al}) / n(\mathrm{M})$ 达到 $1050 \times 10^{-5}$ 时, $\mathrm{AlEtCl}_{2}$ 催化异戊二烯聚合的转化率可达 $75.85 \%(w)$; 而 $\mathrm{AlEt}_{3} 、 \mathrm{Al}(i-\mathrm{Bu})_{3} 、 \mathrm{AlH}(i-\mathrm{Bu})_{2}$ 和 $\mathrm{AlEt}_{2} \mathrm{Cl}$ 催化异戊二 烯聚合的单体转化率依然较低. 从实验结果可以看出, 在低烷基铝浓度下, $\mathrm{AlEt}_{3} 、 \mathrm{Al}(i-\mathrm{Bu})_{3} 、 \mathrm{AlH}(i-\mathrm{Bu})_{2}$ 和 $\mathrm{AlEt}_{2} \mathrm{Cl}$ 催化能力弱, 产率低. 烷基铝引发异成二烯阳离 子聚合的活性与烷基铝在聚合体系中的 Lewis 酸性强弱 有关. 在聚合过程中 $\mathrm{AlEtCl}_{2}$ 的 Lewis 酸性最强, 因此 $\mathrm{AlEtCl}_{2}$ 产生阳离子活性物种的能力较强, 高浓度的 $\mathrm{AlEtCl}_{2}$ 催化异戊二烯的产率较高, 且保持较高的催化 活性(g.P $/ \mathrm{mol} . \mathrm{Al} / \mathrm{h})$.

\section{2 产物结构表征}

产物的 GC-MS 和 ${ }^{1} \mathrm{H}$ NMR 测试谱图如图 2 和图 3 所示. GC-MS 谱图中在流出时间为 5.5 7 $\mathrm{min}$ 检测出异 戊二烯的环状二聚体(图 2 中 e), ${ }^{1} \mathrm{H}$ NMR 中也发现该结 构的环内双键氢 $=\mathrm{CH}$-的化学位移 ( $\delta$ 5.37)(图 3). 图 2 中 $\mathrm{a} \sim \mathrm{d}$ 为流动相及其它溶剂等, 此处不进一步讨论. 对产 物 ${ }^{1} \mathrm{H}$ NMR 谱中各环状二聚体和线型聚合物链的各氢 原子的化学位移进行分析, 分别选取环状二聚体中环内 双键上的 $=\mathrm{CH}-(\delta 5.37)$ 和线型结构聚合物主链双键的 $=\mathrm{CH}-(\delta 5.10)$ 来计算两种结构的相对含量. 同时采用 侧甲基- $\mathrm{CH}_{3}$ 氢计算顺-1,4-结构 $(\delta$ 1.67)和反-1,4-结构 $(\delta$ $1.59)$ 的相对含量. 
表 1 不同烷基铝催化异戊二烯聚合结果汇总

Table 1 Summary of isoprene polymerization catalyzed by different alkylaluminiums

\begin{tabular}{|c|c|c|c|c|c|c|c|c|c|c|c|}
\hline \multirow{2}{*}{$\mathrm{AlR}_{3}$} & \multirow{2}{*}{$\begin{array}{l}n(\mathrm{Al}) / n(\mathrm{M}) \\
\left(\times 10^{-5}\right)\end{array}$} & \multirow{2}{*}{$\begin{array}{l}\text { Conv. } \\
(w / \%)\end{array}$} & \multirow{2}{*}{$\begin{array}{c}\text { Activity } \\
\text { (g.P/mol.Al/h) }\end{array}$} & \multirow{2}{*}{$\begin{array}{c}\text { Linear 1,4- } \\
\text { structured } \\
\text { polymer } \\
(\mathrm{mol} \%)^{a}\end{array}$} & \multirow{2}{*}{$\begin{array}{c}\text { Cyclic } \\
\text { dimer } \\
(\mathrm{mol} \%)^{a}\end{array}$} & \multicolumn{4}{|c|}{$\mathrm{GPC}^{b}$} & \multicolumn{2}{|c|}{$\begin{array}{l}\text { Microstructure of linear } \\
\text { 1,4-polymers }(\mathrm{mol} \%)^{a}\end{array}$} \\
\hline & & & & & & $\begin{array}{l}M_{\mathrm{w} 1}{ }^{c} \\
\left(\times 10^{4}\right)\end{array}$ & $M_{\mathrm{wl}} / M_{\mathrm{n} 1}$ & $\begin{array}{c}M_{\mathrm{w} 2}{ }^{d} \\
\left(\times 10^{4}\right)\end{array}$ & $M_{\mathrm{w} 2} / M_{\mathrm{n} 2}$ & trans $-1,4$ & $c i s-1,4$ \\
\hline \multirow{4}{*}{$\mathrm{AlEt}_{3}$} & 7 & 0.1 & 1946.0 & 35.6 & 64.4 & 0.04 & 1.1 & - & - & 93.9 & 6.1 \\
\hline & 35 & 0.26 & 1011.9 & 36.2 & 63.8 & 0.04 & 1.3 & - & - & 59.1 & 40.9 \\
\hline & 350 & 0.36 & 140.1 & 23.5 & 76.5 & $\begin{array}{c}0.03 \\
(0.05)\end{array}$ & $\begin{array}{c}1 \\
(1.1)\end{array}$ & - & - & 46.0 & 54.0 \\
\hline & 1050 & 2.97 & 385.3 & 49.0 & 51.0 & - & - & 8.8 & 3.6 & 65.9 & 34.1 \\
\hline \multirow{4}{*}{$\mathrm{Al}(i-\mathrm{Bu})_{3}$} & 7 & 0.08 & 1556.8 & 40.7 & 59.3 & 0.05 & 1.1 & - & - & 55.8 & 44.2 \\
\hline & 35 & 0.2 & 778.4 & 41.7 & 58.3 & 0.04 & 1.2 & - & - & 60.5 & 39.5 \\
\hline & 350 & 0.44 & 171.2 & 51.3 & 48.7 & 0.05 & 1.2 & - & - & 37.9 & 62.1 \\
\hline & 1050 & 0.87 & 112.9 & 43.3 & 56.7 & $\begin{array}{c}0.03 \\
(0.05)\end{array}$ & $\begin{array}{c}1 \\
(1.1)\end{array}$ & - & - & 16.6 & 83.4 \\
\hline \multirow{4}{*}{$\mathrm{AlH}(i-\mathrm{Bu})_{2}$} & 7 & 0.06 & 1167.6 & 15.5 & 84.5 & $\begin{array}{c}0.03 \\
(0.06)\end{array}$ & $\begin{array}{c}1 \\
(1.3)\end{array}$ & - & - & 71.8 & 28.2 \\
\hline & 35 & 0.27 & 1050.8 & 5.5 & 94.5 & 0.05 & 1.2 & - & - & 55.4 & 44.6 \\
\hline & 350 & 0.53 & 206.3 & 7.3 & 92.7 & $\begin{array}{c}0.03 \\
(0.05)\end{array}$ & $\begin{array}{c}1 \\
(1.1)\end{array}$ & - & - & 43.6 & 56.4 \\
\hline & 1050 & 0.77 & 99.9 & 41.2 & 58.8 & $\begin{array}{c}0.03 \\
(0.05)\end{array}$ & $\begin{array}{c}1 \\
(1.1)\end{array}$ & - & - & 63.6 & 36.4 \\
\hline \multirow{5}{*}{$\mathrm{AlEt}_{2} \mathrm{Cl}$} & 7 & 0.06 & 1167.6 & 17.2 & 82.8 & $\begin{array}{c}0.02 \\
(0.05)\end{array}$ & $\begin{array}{c}1 \\
(1.2)\end{array}$ & 11.9 & 2.8 & 90.3 & 9.7 \\
\hline & 35 & 0.33 & 1284.4 & 4.2 & 95.8 & - & - & 8.7 & 2.9 & 77.8 & 22.2 \\
\hline & 350 & 0.43 & 167.4 & 8.4 & 91.6 & $\begin{array}{c}0.03 \\
(0.06)\end{array}$ & $\begin{array}{c}1 \\
(1.2)\end{array}$ & - & - & 73.7 & 26.3 \\
\hline & 1050 & 2.56 & 332.1 & 65.8 & 34.2 & 0.06 & 1.2 & 1.6 & 2.2 & 6.3 & 93.8 \\
\hline & 7 & 0.41 & 7978.6 & 74.1 & 25.9 & 0.04 & 1.1 & - & - & 75.9 & 24.1 \\
\hline \multirow[t]{3}{*}{$\mathrm{AlEtCl}_{2}$} & 35 & 0.37 & 1440.0 & 66.7 & 33.3 & $\begin{array}{c}0.03 \\
(0.06)\end{array}$ & $\begin{array}{c}1 \\
(1.1)\end{array}$ & 21.5 & 5.8 & 74.1 & 25.9 \\
\hline & 350 & 0.81 & 315.3 & 38.6 & 61.4 & 0.9 & 1.5 & 32.7 & 2.9 & 70.6 & 29.4 \\
\hline & 1050 & 75.58 & 9805.2 & 82.0 & 18.0 & 0.03 & 1 & 0.3 & 5.4 & 71.1 & 28.9 \\
\hline
\end{tabular}

Conditions: $n(\mathrm{Al}) / n(\mathrm{M})=7 \times 10^{-5}, 35 \times 10^{-5}, 350 \times 10^{-5}, 1050 \times 10^{-5} ; T=30{ }^{\circ} \mathrm{C} ; t=30 \mathrm{~min} .{ }^{a}$ From ${ }^{1} \mathrm{H}$ NMR, ${ }^{b}$ from GPC, ${ }^{c}$ from low molecular weight peak of the GPC curve of the product, ${ }^{d}$ from high molecular weight peak of the GPC curve of the product.

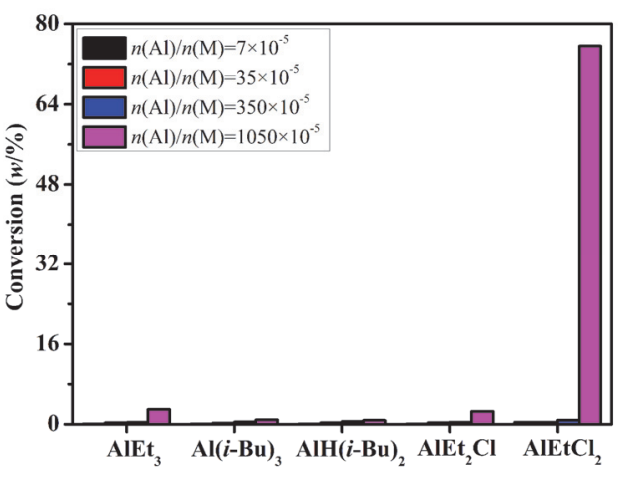

Alkylaluminiums

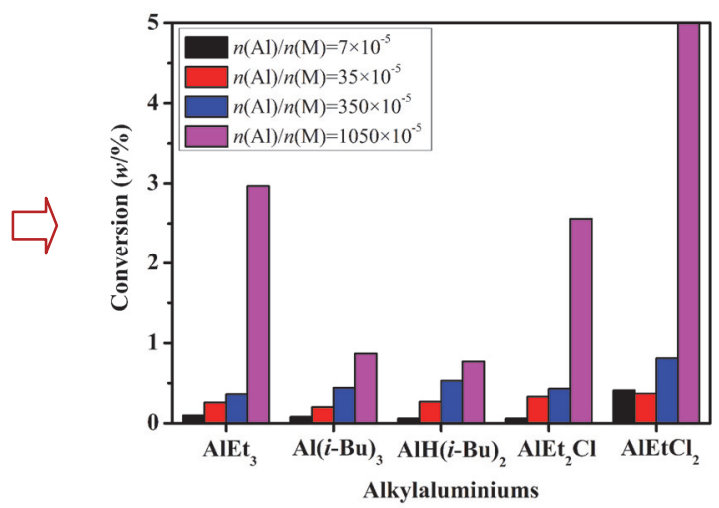

图 1 不同烷基铝催化异戊二烯聚合的转化率

Figure 1 Conversion of isoprene catalyzed by different alkylaluminiums

线型聚合物和环状二聚体的相对含量计算, 见公式 (1):

$$
C_{\text {linear 1,4-structured polymer }}=\frac{A_{5.10}}{A_{5.10}+A_{5.37}}
$$

线型结构聚合物中顺-1,4-和反-1,4-结构相对含量 计算，见公式(2,3):

$$
C_{\text {trans }-1,4}=\frac{\frac{1}{3} A_{1.59}}{\frac{1}{3} A_{1.59}+\frac{1}{3} A_{1.67}}
$$



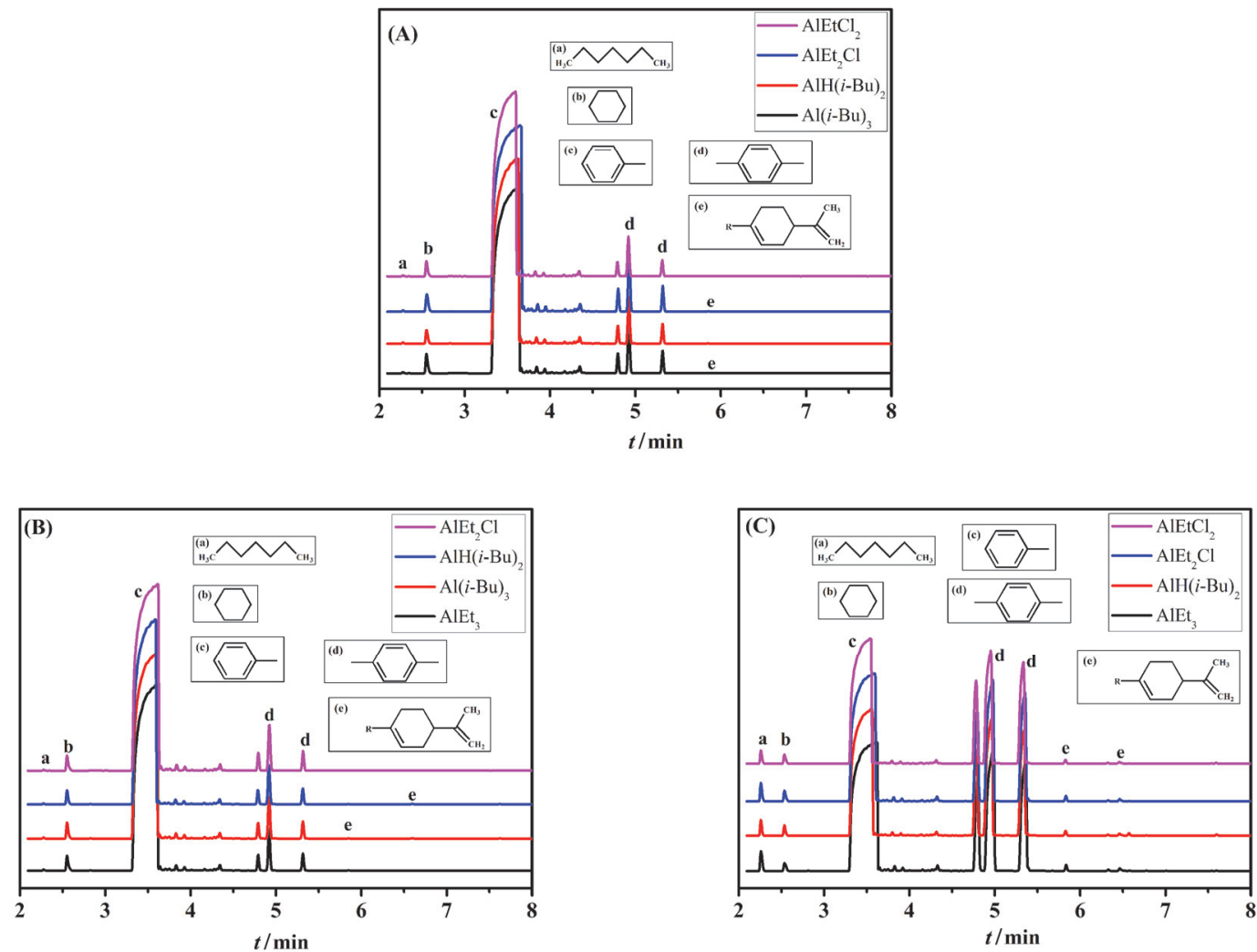

图 2 不同烷基铝催化产物的 GC-MS 谱图

Figure 2 GC-MS spectra of products from different alkylaluminiums

(A) $30{ }^{\circ} \mathrm{C}, n(\mathrm{Al}) / n(\mathrm{M})=35 \times 10^{-5}$; (B) $30{ }^{\circ} \mathrm{C}, n(\mathrm{Al}) / n(\mathrm{M})=350 \times 10^{-5}$; (C) $30{ }^{\circ} \mathrm{C}, n(\mathrm{Al}) / n(\mathrm{M})=1050 \times 10^{-5}$
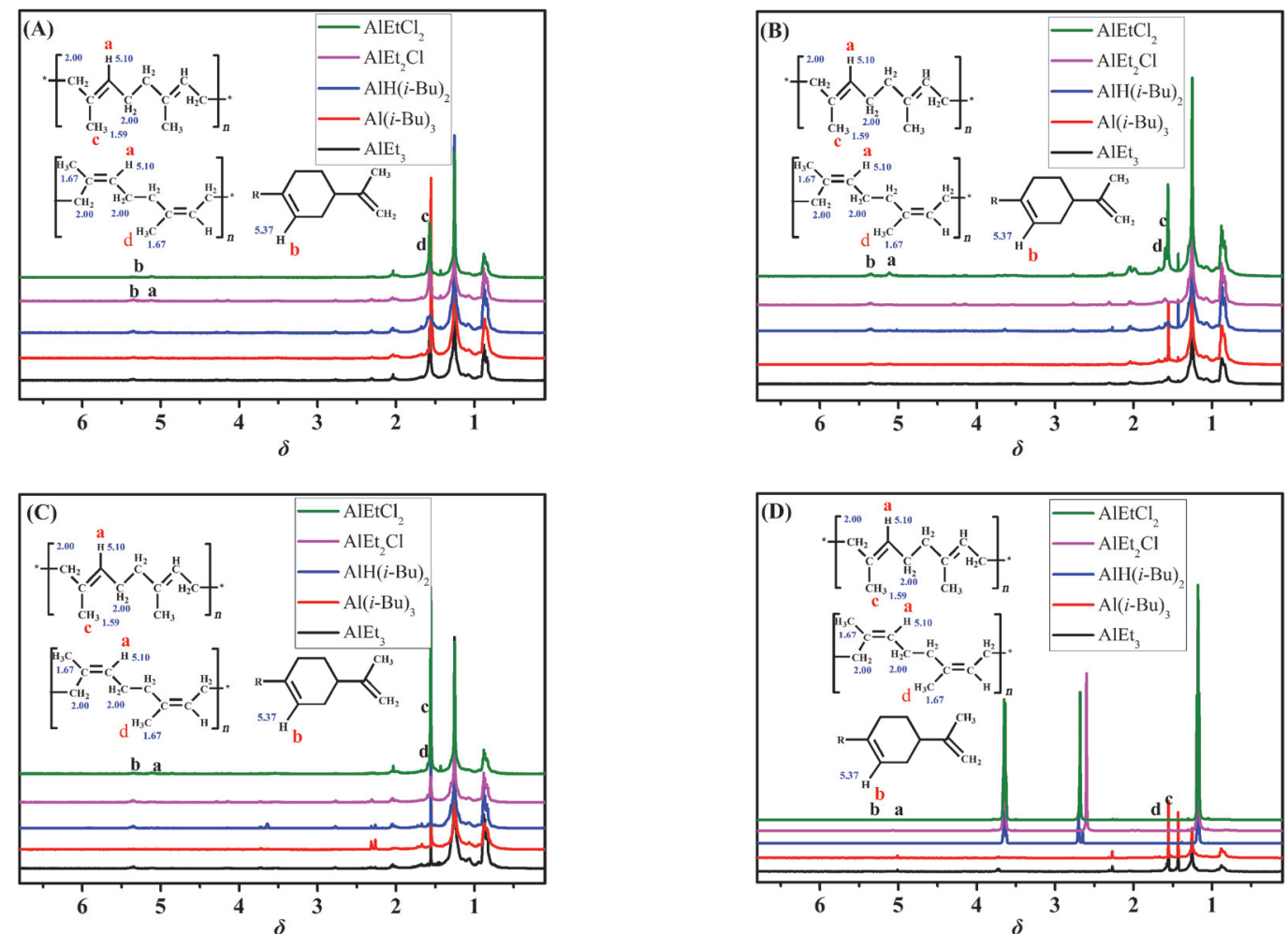

图 3 不同烷基铝催化产物的 ${ }^{1} \mathrm{H}$ NMR 谱图

Figure $3{ }^{1} \mathrm{H}$ NMR spectra of the products from different alkylaluminiums

(A) $30{ }^{\circ} \mathrm{C}, n(\mathrm{Al}) / n(\mathrm{M})=7 \times 10^{-5}$; (B) $30{ }^{\circ} \mathrm{C}, n(\mathrm{Al}) / n(\mathrm{M})=35 \times 10^{-5}$; (C) $30{ }^{\circ} \mathrm{C}, n(\mathrm{Al}) / n(\mathrm{M})=350 \times 10^{-5}$; (D) $30{ }^{\circ} \mathrm{C}, n(\mathrm{Al}) / n(\mathrm{M})=1050 \times 10^{-5}$ 


$$
C_{c i s-1,4}=\frac{\frac{1}{3} A_{1.67}}{\frac{1}{3} A_{1.59}+\frac{1}{3} A_{1.67}}
$$

图 4 为根据公式(1)计算所得 $30{ }^{\circ} \mathrm{C}$ 下不同烷基铝催 化异戊二烯聚合产物的线型 1,4-结构和环状二聚体的相 对含量. 当 $n(\mathrm{Al}) / n(\mathrm{M}) \leqslant 350 \times 10^{-5}$ 时, $\mathrm{AlEt}_{3} 、 \mathrm{Al}(i-\mathrm{Bu})_{3}$ 、 $\mathrm{AlH}(i-\mathrm{Bu})_{2}$ 和 $\mathrm{AlEt}_{2} \mathrm{Cl}$ 更倾向于催化异戊二烯形成环状 二聚体，而 $\mathrm{AlEtCl}_{2}$ 更易催化异戊二烯聚合为线性聚合 物; 当 $n(\mathrm{Al}) / n(\mathrm{M})=1050 \times 10^{-5}$ 时, 烷基铝催化异戊二 烯的线性聚合物含量明显增加, 环状二聚体含量降低. 说明烷基铝浓度提高以后, 异戊二烯发生阳离子型链增 长(聚合)的倾向提高, 而催化形成环状齐聚物的倾向降 低.

根据公式(2), (3)计算得到线性聚合物中顺式-1,4 和 反式-1,4 结构含量(图 5). 五种烷基铝催化所得聚异戊二 烯均为顺反混合结构, 其中 $\mathrm{AlEt}_{3} 、 \mathrm{Al}(i-\mathrm{Bu})_{3} 、 \mathrm{AlH}(i-\mathrm{Bu})_{2}$ 和 $\mathrm{AlEt} \mathrm{t}_{2} \mathrm{Cl}$ 催化合成的聚合物中顺式-1,4 结构和反式-1,4 结构的相对含量无明显变化规律; 而在 $\mathrm{AlEtCl}_{2}$ 催化合 成的聚合物中反式 $-1,4$ 结构的含量占 $70 \%$ 以上. 综上所 述, 烷基铝催化异成二烯聚合时, 立构规整选择能力差.

$30{ }^{\circ} \mathrm{C}$ 下不同烷基铝催化异戊二烯齐聚与聚合产物 的分子量及分子量分布列于表 1 . 从表 1 可以发现, 不 含氯的烷基铝催化产物的分子量绝大部分都在 300
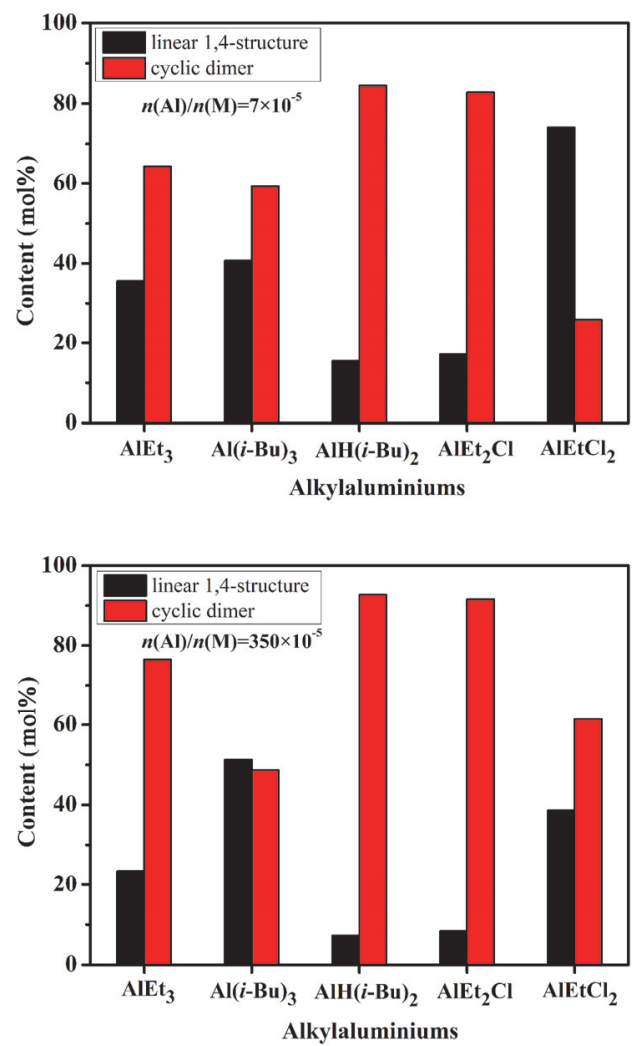

图 4 线性聚合物和环状二聚体的相对含量

Figure 4 Relative content of linear polymers and cyclic dimers
600 的范围内, 且分子量的分布很窄 $\left(M_{\mathrm{w}} / M_{\mathrm{n}}=1 \sim 2\right)$, 说 明其产物为低分子量齐聚物 (主要为环状低聚物). $\mathrm{AlEt}_{2} \mathrm{Cl}$ 和 $\mathrm{AlEtCl}_{2}$ 这类阳离子聚合活性更强的烷基铝, 除了齐聚物组分, 存在较明显的较高分子量的线性聚合 物组分. 在图 6 中, 发现烷基铝浓度过低 $(n(\mathrm{Al}) / n(\mathrm{M})=7$ $\times 10^{-5}$ )时，烷基铝催化异戊二烯齐聚倾向增大，低聚物 含量较高. 对阳离子活性较高的烷基铝 $\left(\mathrm{AlEt}_{2} \mathrm{Cl}\right.$ 和 $\mathrm{AlEtCl}_{2}$ )而言，高烷基铝浓度可获得更高含量的线性聚 合物, 但由于链转移作用, 线性聚合物的分子量显著降 低. 但阳离子活性较弱的 $\mathrm{AlEt}_{3}$ 在高烷基铝浓度下，阳 离子聚合倾向增大, 线性聚合物中高分子量组分含量增 大(图 6d).

\section{3 烷基铝引发异戊二烯齐聚与聚合机理}

在聚合体系中，两分子的烷基铝可能形成缔合结 构, 缔合的烷基铝分子可以可逆地解离为阴离子 $\left(\left[\mathrm{R}_{4} \mathrm{Al}\right]^{-},\left[\mathrm{RAlCl}_{3}\right]^{-}\right.$等 $)$和阳离子 $\left(\left[\mathrm{R}_{2} \mathrm{Al}\right]^{+},[\mathrm{RAlCl}]^{+}\right.$

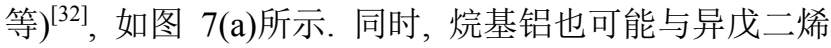
单体中的微量水(单体含水量 $0.0065 \%$ )作用 ${ }^{[26]}$, 解离得 到阴、阳离子(图 7(b)). 这两种解离过程可能同时存在. 解离的程度受到烷基铝的种类、浓度等影响. $\mathrm{Al}(i-\mathrm{Bu})_{3}$ 和 $\mathrm{AlH}(i-\mathrm{Bu})_{2}$ 由于较大的烷基位阻, 不易解离, 所以阳 离子聚合活性极弱. 另外, 提高烷基铝浓度, 其活性有 所降低, 说明烷基铝浓度提高并不能提高其解离程度和 活性.
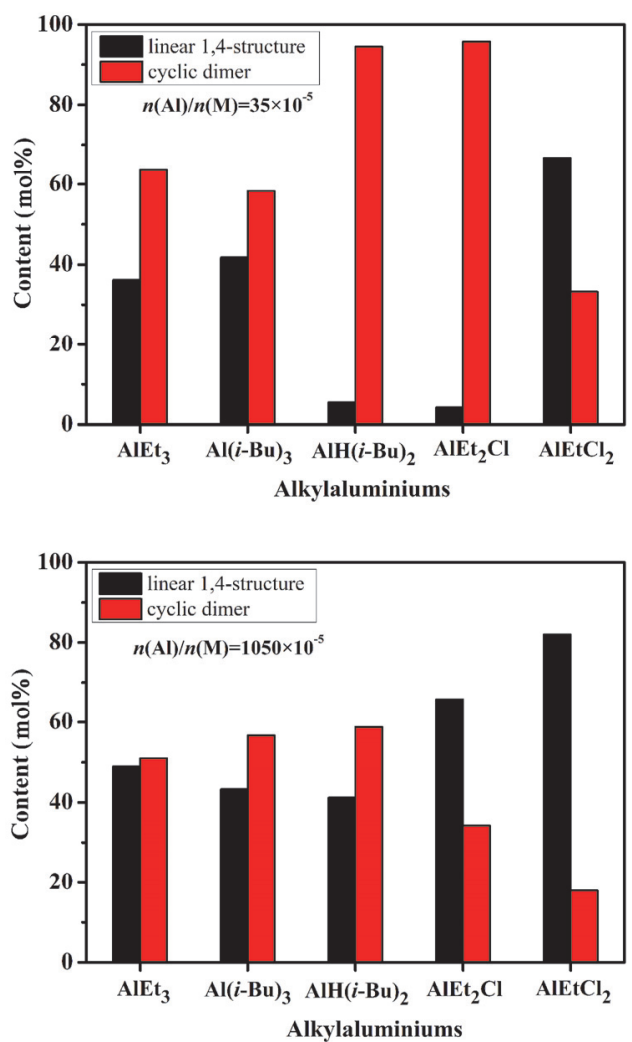

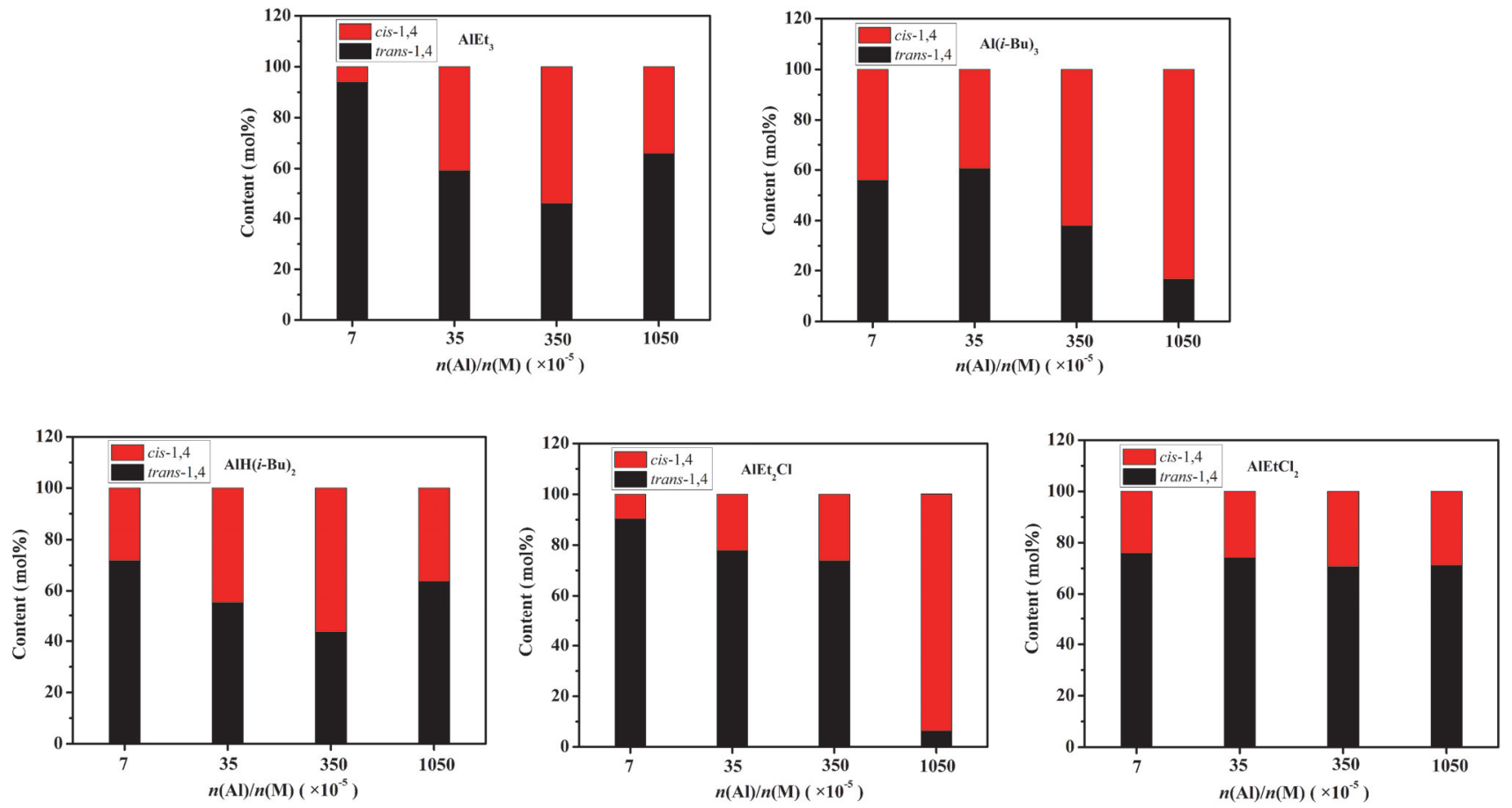

图 5 线性聚合物的微观结构

Figure 5 The microstructures of linear polymers
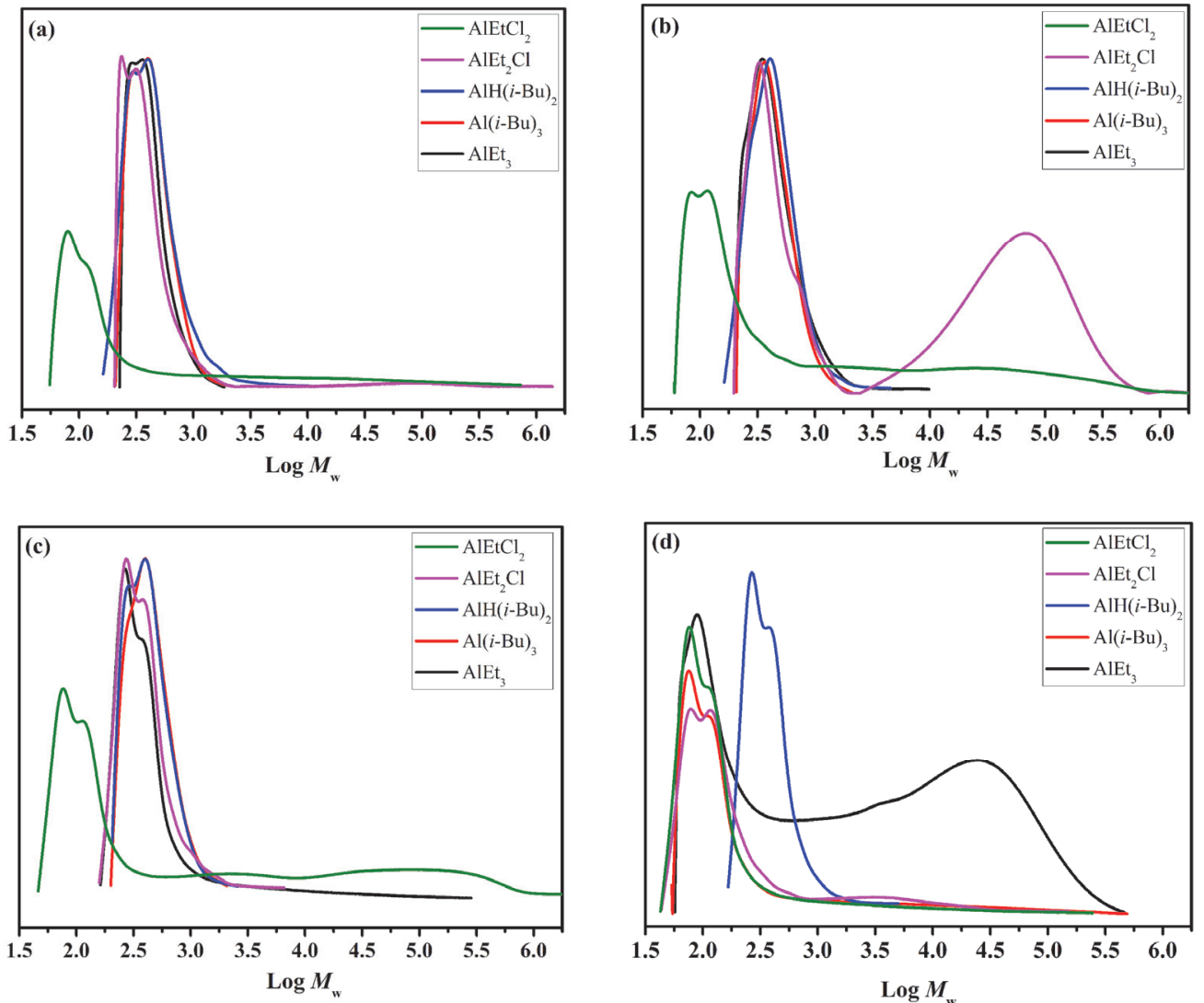

图 6 不同烷基铝催化产物的 GPC 微分曲线

Figure 6 Differential curves of GPC of products catalyzed by different alkylaluminiums

(a) $30{ }^{\circ} \mathrm{C}, n(\mathrm{Al}) / n(\mathrm{M})=7 \times 10^{-5}$; (b) $30{ }^{\circ} \mathrm{C}, n(\mathrm{Al}) / n(\mathrm{M})=35 \times 10^{-5}$; (c) $30{ }^{\circ} \mathrm{C}, n(\mathrm{Al}) / n(\mathrm{M})=350 \times 10^{-5}$; (d) $30{ }^{\circ} \mathrm{C}, n(\mathrm{Al}) / n(\mathrm{M})=1050 \times 10^{-5}$ 
(a)

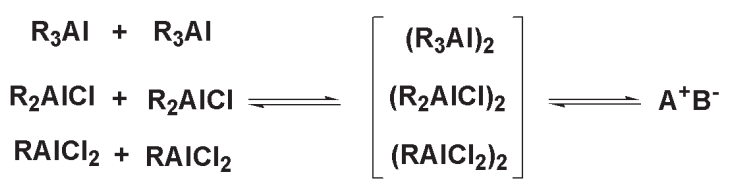

(b)

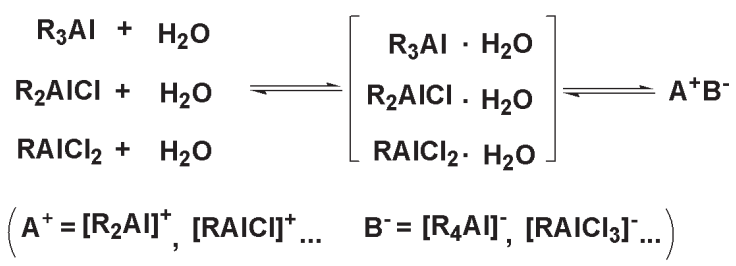

图 7 烷基铝产生阴阳离子的可能方式

Figure 7 Possible ways for alkylaluminium to produce anions and cations

烷基铝解离产生的阴阳离子在一定程度上可以诱 导异戊二烯单体离子化(图 8), 当异戊二烯单体被诱导 产生阳离子后, 一方面(过程 I), 异戊二烯分子间可以发 生分子内电荷转移而形成环状二聚体(或环状齐聚物); 另一方面(过程 II), 异戊二烯单体被诱导产生阳离子后 也可能继续进行阳离子型链增长, 形成高分子量聚合 物. 阳离子型反应 I 和 II 分别是异戊二烯的主要齐聚和 聚合过程.

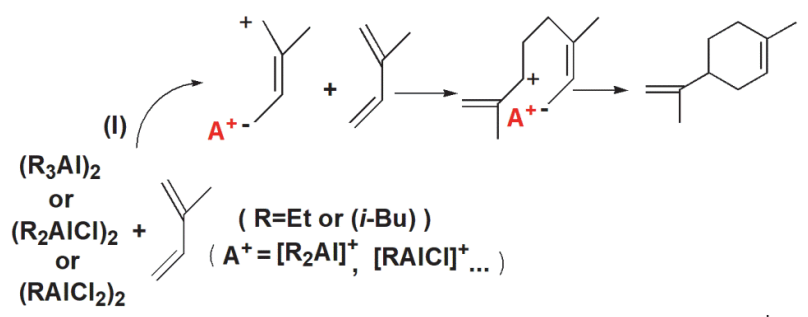

(II) $\backslash[\mathrm{AII} \uparrow$

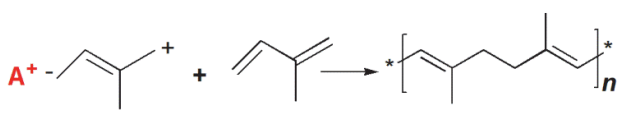

图 8 不同烷基铝催化异戊二烯聚合获得环二聚体和线性聚合物的可 能机理

Figure 8 Possible mechanism of synthesis of cyclic dimers and linear polymers by using different alkylaluminium compounds

$\mathrm{AlEtCl}_{2}$ 产生阳离子活性物种的能力较强, 阳离子 聚合活性较强, 催化异戊二烯聚合时更倾向(II)方向进 行, 得到分子量较高的线性聚合物; $\mathrm{Al}(i-\mathrm{Bu})_{3}$ 和 $\mathrm{AlH}(i-\mathrm{Bu})_{2}$ 的阳离子聚合活性极弱, 主要引发异戊二烯 齐聚得到环状齐聚物(I). 两种反应同时存在, 对于阳离 子活性较弱的烷基铝, 适当提高烷基铝的浓度, 可能增 大向反应(II)方向进行的几率, 获得高分子量聚合物(如 高浓度 $\mathrm{AlEt}_{3}$ 催化异戊二烯聚合的产物中出现高分子量 组分). 但对阳离子活性较高的烷基铝(如 $\mathrm{AlEtCl}_{2}$ ), 烷基 铝浓度过高, 虽然催化异戊二烯聚合时更倾向(II)方向 进行, 但由于链转移作用, 线性聚合物的分子量显著降 低.

\section{3 结论}

使用单组分烷基铝 $\left(\mathrm{AlEt}_{3} 、 \mathrm{Al}(i-\mathrm{Bu})_{3} 、 \mathrm{AlH}(i-\mathrm{Bu})_{2}\right.$ 、 $\mathrm{AlEt}_{2} \mathrm{Cl}$ 和 $\mathrm{AlEtCl}_{2}$ ) 催化异戊二烯聚合, 得到的产物中包 含低分子量的齐聚物(主要为环二聚体)和高分子量的线 性聚合物. 当 $n(\mathrm{Al}) / n(\mathrm{M}) \leqslant 350 \times 10^{-5}$ 时, $\mathrm{AlEt}_{3}$ 、 $\mathrm{Al}(i-\mathrm{Bu})_{3} 、 \mathrm{AlH}(i-\mathrm{Bu})_{2} 、 \mathrm{AlEt}_{2} \mathrm{Cl}$ 和 $\mathrm{AlEtCl}_{2}$ 的催化活性均 极低; 当 $n(\mathrm{Al}) / n(\mathrm{M})=1050 \times 10^{-5}$ 时, $\mathrm{AlEtCl}_{2}$ 的催化活 性显著提高, 转化率可达 $75.58 \%(w)$, 且所得产物主要 为 1,4-结构为主的线性聚合物. 这是因为 $\mathrm{AlEtCl}_{2}$ 产生 阳离子活性物种的能力较强, 可获得更多活性物种和更 高的反应活性(g.P $/ \mathrm{mol} . \mathrm{Al} / \mathrm{h})$. 同时由于阳离子聚合时极 易发生链转移, 较高的烷基铝浓度也不利于获得高分子 量的聚合物.

因此, 采用 Ziegler-Natta 催化体系催化异戊二烯聚 合时, 若 $n(\mathrm{Al}) / n(\mathrm{M}) \leqslant 350 \times 10^{-5}$, 烷基铝自身引发异戊 二烯齐聚及阳离子聚合的能力极弱, 对配位聚合体系的 影响较小.

\section{4 实验部分}

\section{1 试剂}

异戊二烯(Ip), 聚合级, 山东华聚高分子材料有限 公司提供，使用前减压蒸馏精制，单体含水量 $0.0065 \%$; $\mathrm{AlEt}_{3} 、 \mathrm{Al}(i-\mathrm{Bu})_{3} 、 \mathrm{AlH}(i-\mathrm{Bu})_{2}$ 由山东华聚高分子材料有 限公司提供, $\mathrm{AlEt}_{2} \mathrm{Cl}$ 和 $\mathrm{AlEtCl}_{2}$ 购于百灵威科技有限公 司; 正庚烷购于天津市富宇精细化工有限公司, 使用前 采用金属钠回流精制.

\section{2 聚合}

$100 \mathrm{~mL}$ 圆底烧瓶洗净、干燥、组装, 抽排、 $\mathrm{N}_{2}$ 置 换三次，按照聚合条件，依次向反应瓶中加入定量的精 制单体、烷基铝后实施聚合. 当烷基铝为 $\mathrm{AlEt}_{3}$ 、 $\mathrm{Al}(i-\mathrm{Bu})_{3} 、 \mathrm{AlH}(i-\mathrm{Bu})_{2} 、 \mathrm{AlEt}_{2} \mathrm{Cl}$ 时实施本体聚合, 当烷 基铝为 $\mathrm{AlEtCl}_{2}$ 时以正庚烷为溶剂实施溶液聚合(单体 浓度为 $30 \%(w))$. 恒温聚合一定时间(30 min)后加入 2 $5 \mathrm{~mL}$ 酒精终止反应, 样品于通风橛中挥发干燥, 并进一 步真空干燥至恒重.

\section{3 仪器表征}

${ }^{1} \mathrm{H}$ NMR 测试采用 AVANCE III $500 \mathrm{MHz}$ 型核磁 共振仪(Bruker 公司), 氝代氯仿 $\left(\mathrm{CDCl}_{3}\right)$ 为溶剂, 四甲基 硅烷为内标, 测试温度 $25{ }^{\circ} \mathrm{C}$.

产物的 GPC 测试采用 HLC-8320 型凝胶渗透色谱仪 (日本 TOSOH 株式会社), 四氢呋喃(THF)为溶剂, $40{ }^{\circ} \mathrm{C}$, 聚苯乙烯为标样. 通过 GPC 曲线获得产物中不同组分 的分子量.

GC-MS 采用 Agilent 5977B GC/MSD 型气相色谱/ 质谱联用仪测试. 甲苯为溶剂, 电子电离, 液化加热器 于 $250{ }^{\circ} \mathrm{C}$ 将液体液化, 分流比 $100 ： 1$. 柱箱: (1) $50{ }^{\circ} \mathrm{C}$ 
保持 $2 \mathrm{~min}$, (2) 加热至 $150{ }^{\circ} \mathrm{C}$, 速率为 $10{ }^{\circ} \mathrm{C} / \mathrm{min}$, 在 $150{ }^{\circ} \mathrm{C}$ 下保持 $1 \mathrm{~min}$, (3) 加热至 $200{ }^{\circ} \mathrm{C}$, 速率为 $50{ }^{\circ} \mathrm{C} /$ $\mathrm{min}$, 在 $200{ }^{\circ} \mathrm{C}$ 处保持 $3 \mathrm{~min}$. 产物用甲苯溶解后, 过滤, 对甲苯溶液进行测试.

单体水含量测试采用 SF101 型微量水分测定仪(淄 博淄分仪器有限公司). 测试时用 $50 \mu \mathrm{L}$ 注射器准确量取 $10 \mu \mathrm{L}$ 精制后的异戊二烯, 通过进样口注入微量水分测 定仪电解瓶液面以下进行测试, 测试三次取平均值.

\section{References}

[1] Mori, H.; Hasebe, K.; Terano, M. Polymer 1999, 40, 1389.

[2] Yang, H. R.; Zhang, L. T.; Zang, D. D.; Fu, Z. S.; Fan, Z. Q. Catal. Commun. 2015, 62, 104.

[3] Bahri-Laleh, N.; Correa, A.; Mehdipour-Ataei, S.; Arabi, H.; Haghighi, M. N.; Zohuri, G.; Cavallo, L. Macromolecules 2011, 44, 778.

[4] Liu, B. P.; Nitta, T.; Nakatani, H.; Terano, M. Macromol. Chem. Phys. 2002, 203, 2412.

[5] Liu, B. P.; Nitta, T.; Nakatani, H.; Terano, M. Macromol. Chem. Phys. 2003, 204, 395.

[6] Liu, B. P.; Nitta, T.; Nakatani, H.; Terano, M. Macromol. Symp. 2004, 213, 7 .

[7] Potapov, A. G.; Terskikh, V. V.; Zakharov, V. A.; Bukatov, G. D. J. Mol. Catal. A: Chem. 1999, 145, 147.

[8] Potapov, A. G.; Terskikh, V. V.; Bukatov, G. D.; Zakharov, V. A. J. Mol. Catal. A: Chem. 2000, 158, 457.

[9] Hu, J.; Han, B.; Shen, X. R.; Fu, Z. S.; Fan, Z. Q. Chin. J. Polym. Sci. 2013, 31, 583.

[10] Franceschini, F. C.; Tavares, T. T. D. R.; Greco, P. P.; Galland, G. B.; dos Santos, J. H.; Soares, J. B. J. Appl. Polym. Sci. 2005, 95, 1050.

[11] Rocha, T. C. J.; Coutinho, F. M. B.; Soares, B. G. Polym. Bull. 2009, $62,1$.

[12] Gao, H.; Liu, X.; Tang, Y.; Pan, J.; Wu, Q. Polym. Chem. 2011, 2, 1398.
[13] Huang, W. Q.; Yang, L.; Zhao, X.; Man, Y.; Li, B. Y.; Zhang, Y.; Yang, W. T. Chem. Ind. \& Eng. Pro. 2011, 30, 1231 (in Chinese). (黄 文氢, 杨岭, 赵曦, 满毅, 李秉毅, 张颖, 杨万泰, 化工进展, 2011, 30, 1231.)

[14] Blaakmeer, E. S. M.; van Eck, E. R. H.; Kentgens, A. P. M. Phys. Chem. Chem. Phys. 2018, 20, 7974.

[15] Niu, Q. T.; Zhang, J. Y.; Peng, W.; Fan, Z. Q.; He, A. H. Mol. Catal. 2019, $471,1$.

[16] Richardson, W. S. J. Polym. Sci. 1954, 13, 325.

[17] Ferington, T. E.; Tobolsky, A. V. J. Polym. Sci. 1958, 31, 25.

[18] Dolgoplosk, B.; Belonovskaia, G. P.; Boldyreva, I. I.; Nelson, K. V.; Kropacheva, E. N.; Rosinoer, J. M.; Chernova, J. D. J. Polym. Sci. 1961, 53, 209.

[19] Kennedy, J. P.; Squires, R. G. Polymer 1965, 6, 579.

[20] Binder, J. L. J. Polym. Sci. Part A: Gen. Pap. 1963, 1, 37.

[21] Kössler, I.; Vodehnal, J.; Štolka, M. J. Polym. Sci. Part A: Gen. Pap. 1965, 3, 2081.

[22] Pétiaud, R.; Taärit, Y. B. J. Chem. Soc. 1980, 10, 1385.

[23] Peng, Y. X.; Deng, Y. G.; Liu, J. L.; Dai, H. S.; Cun, L. F. Polym. Mater. Sci. Eng. 1997, 13, 95 (in Chinese). (彭宇行, 邓勇刚, 刘佳 林, 戴汉松, 寸琳峰, 高分子材料科学与工程, 1997, 13, 95.)

[24] Gaylord, N. G.; Matyska, B.; Mach, K.; Vodehnal, J. J. Polym. Sci. Pol. Chem. 1966, 4, 2493.

[25] Gaylord, N. G.; Kössler, I.; Matyska, B.; Mach, K. J. Polym. Sci. Pol. Chem. 1968, 6, 125.

[26] Matyska, B.; Doležal, I.; Kössler, I. Collect. Czech. Chem. C 1971 36, 2924.

[27] Uchida, Y.; Furuhata, K. I.; Ishiwata, H. Bull. Chem. Soc. Jpn. 1971, 44, 1118.

[28] Akutagawa, S.; Taketomi, T.; Otsuka, S. Chem. Lett. 1976, 5, 485.

[29] Shen, G. L. Journal of Liaoyang Petrochemical College 1996, 12, 1 (in Chinese). (沈国良, 辽阳石油化专学报, 1996, 12, 1.)

[30] Yu, S. J. Speciality Petrochemicals 2000, (2), 20 (in Chinese). (于士 君, 精细石油化工, 2000, (2), 20.)

[31] Shen, G. L.; Tang, L. H. Speciality Petrochemicals 2004, (1), 17 (in Chinese). (沈国良, 唐丽华, 精细石油化工, 2004, (1), 17.)

[32] Xia, S. W.; Xia, S. W.; Ma, S. X. Acta Polym. Sinica 1998, (3), 262 (in Chinese). (夏树伟, 夏少武, 马世学, 高分子学报, 1998, (3), 262.)

(Cheng, B.) 